DOI: $10.15593 / 2224-9877 / 2018.1 .06$

УДК 621.9

\author{
Т.Р. Абляз ${ }^{1}$, К.Р. Муратов ${ }^{1}$, Е.Е. Красновский ${ }^{2}$, Д.А. Борисов ${ }^{1}$ \\ ${ }^{1}$ Пермский национальный исследовательский политехнический университет, Пермь, Россия \\ ${ }^{2}$ Московский государственный технический университет им. Н.Э. Баумана \\ (национальный исследовательский университет), Москва, Россия

\section{МОДЕЛИРОВАНИЕ ТЕМПЕРАТУРНОГО ВОЗДЕЙСТВИЯ ЕДИНИЧНОГО ИМПУЛЬСА ПРИ ЭЛЕКТРОЭРОЗИОННОЙ ОБРАБОТКЕ}

\begin{abstract}
Целью работы является разработка теоретической модели, позволяющей описать величину температурного воздействия на поверхность заготовки при электроэрозионной обработке. Моделирование единичного импульса осуществлялось с помощью пакета прикладного программного обеспечения Comsol Multyphysics 4.4. При проведении расчетов в качестве материала заготовки выбрана сталь 38X2Н2MА по ГОСТ 4543-71. Моделирование плазменного канала осуществлялось с применением фризики Heat Transfer in Solids. Ввиду того, что канал разряда имеет цилиндрическую форму, моделирование проводилось для двумерной осесимметричной модели. В связи с тем, что легирующие элементы стали 38Х2Н2МА влияют на ее критические точки, в теоретическую модель был внесен поправочный коэфффициент. При моделировании теплового потока учитывалось импульсное воздействие тока на поверхность заготовки путем использования функции Step. B результате моделирования установлено, что температура на поверхности детали в центре разряда в процессе ЭЭО превышает $2500^{\circ}$, в результате чего на обработанной поверхности формируется единичная лунка. Показано, что единичная лунка, полученная в результате моделирования, имеет чашеобразную форму, т.е. ширина кратера выше по сравнению с глубиной, что не противоречит существующим научным данным. Ширина и глубина кратера в диапазоне времени $t=100 \ldots 200$ мкс увеличиваются. При достижении времени $t=200$ мкс температура в лунке становится пиковой, затем она понижается. Показано, что для повышения точности моделирования температурного воздействия на поверхность заготовки при ЭЭО необходимо учитывать импульсный характер воздействия тока и вводить в систему уравнений функцию Step.

Ключевые слова: электроэрозионная обработка, электрод-инструмент, точность, качество, моделирование,
\end{abstract} импульс, температура, напряжение, сила тока, режимы обработки.

\author{
T.R. Ablyaz ${ }^{1}$, K.R. Muratov ${ }^{1}$, E.E. Krasnovsky², D.A. Borisov ${ }^{1}$ \\ ${ }^{1}$ Perm National Research Polytechnic University, Perm, Russian Federation \\ ${ }^{2}$ Bauman Moscow State Technical University (National Research University of Technology), \\ Moscow, Russian Federation
}

\title{
SIMULATION OF THE TEMPERATURE EFFECT OF A SINGLE PULSE DURING ELECTRICAL DISCHARGE MACHINING
}

The aim of the work is to develop a theoretical model that allows one to describe the magnitude of the temperature effect on the workpiece surface during EDM processing. Simulation of a single pulse was carried out using the software package Comsol Multyphysics 4.4. When carrying out calculations, steel 38X2H2MA according to GOST 4543-71 was chosen as the material of the billet. The simulation of the plasma channel was carried out using the physics of "Heat Transfer in Solids". In view of the fact that the discharge channel has a cylindrical shape, the simulation was carried out for a two-dimensional axisymmetric model. Due to the fact that the alloying elements of steel 38X2N2MA influence its critical points, a correction factor has been introduced into the theoretical model. When modeling the heat flow, the impulse effect of the current on the workpiece surface was taken into account by using the "Step" function. As a result of the simulation, it is established that the temperature on the surface of the part in the center of the discharge during the EDM exceeds 2500 degrees, as a result of which a single well is formed on the treated surface. It is shown that the shape of a single well, obtained as a result of modeling, is a cup-like shape, that is, the crater width is higher compared to the depth, which does not contradict the existing scientific data. The width and depth of the crater in the time range $t=100 \ldots 200 \mu \mathrm{s}$ increase. When the time reaches $t=200 \mu \mathrm{s}$, the temperature in the well becomes peak, then it decreases. It is shown that in order to improve the accuracy of simulation of the temperature effect on the surface of the billet in the EEE, it is necessary to take into account the impulse nature of the current effect and introduce the "Step" function into the system of equations.

Keywords: electrical discharge machining, electrode-tool, accuracy, quality, simulation, impulse, temperature, voltage, amperage, processing modes. 
В процессе электроэрозионной обработки (ЭЭО) поверхность обрабатываемого материала подвергается интенсивному тепловому и ударному воздействию. В результате такого воздействия на поверхности заготовки возникают структурные изменения [1-6]. С целью прогнозирования выходных показателей качества обработанной поверхности необходимо определить величину температурного воздействия в процессе ЭЭО.

Для оценки величины температурного воздействия на поверхность заготовки в процессе ЭЭО широкое применение находят методы математического моделирования [7-12]. Рассматриваемые модели с высокой точностью позволяют оценить влияние режимных параметров ЭЭО на формирование показателей качества обработанной поверхности, однако не в полной мере учитывают влияние физико-механических свойств обрабатываемых материалов и импульсный характер воздействия заряда.

В работах [1-3] показано, что на обрабатываемость материалов электроэрозионным способом оказывают влияние теплофизические свойства материалов. Чем выше температура плавления материала, тем меньше материала будет удалено единичным разрядом. Таким образом, электроэрозионная обрабатываемость различных материалов будет характеризоваться удельным расходом энергии на единицу массы снятого материала.

При моделировании процесса ЭЭО необходимо учитывать, что интенсивность эрозионного съема материала зависит от длительности теплового воздействия. При одной и той же энергии разрядного импульса эрозионный съем увеличивается с увеличением длительности разрядного импульса до некоторых пределов, а затем начинает падать [3, 13]. Объясняется это тем, что увеличение длительности воздействия приводит к расширению зоны плавления большого объема материала. Однако при этом снижается плотность энергии разрядного импульса, а следовательно, снижается температура в точках приложения разряда на поверхности электродов.

Целью работы является разработка теоретической модели, позволяющей описать величину температурного воздействия на поверхность заготовки при электроэрозионной обработке.

\section{Материалы и методы исследования}

Моделирование единичного импульса осуществлялось с помощью пакета прикладного программного обеспечения Comsol Multyphysics 4.4.

При проведении расчетов в качестве материала заготовки выбрана сталь 38Х2Н2МА по ГОСТ 4543-71. Заготовка выполнена в виде пластинки размером $10 \times 20 \times 40$ мм. В качестве диэлектрической жидкости использовано трансформаторное масло ГОСТ 982-80. В качестве электрода-инструмента использован медный цилиндр (М3 ГОСТ 859-2001) диаметром 10 мм.

На поверхность детали воздействует плазменный канал разряда, который образуется в результате электрического пробоя диэлектрика. Моделирование осуществлялось с применением физики Heat Transfer in Solids (нагрев твердых тел).

Расчетная схема воздействия канала плазмы на поверхность обрабатываемой детали представлена на рис. 1 [7].

Ввиду того, что канал разряда имеет цилиндрическую форму, моделирование проводилось для двумерной осесимметричной модели. В данных условиях целесообразно использовать цилиндрическую систему координат.

Излучение плазмы испаряет и ионизирует тонкий слой окружающей жидкости, идущий на увеличение массы плазмы. Радиус плазмы увеличивается со временем из-за высокого внутреннего давления в канале и преобразования окружающего диэлектрика в плазму. На это преобразование уходит $70 \%$ общей энергии [2, 7].

Во время расширения канала плазмы его внутреннее давление падает, тогда как давление в прилегающем диэлектрике повышается. За счет короткой продолжительности импульса влияние этого явления можно не учитывать из-за инерционности диэлектрика в целом [7]. 


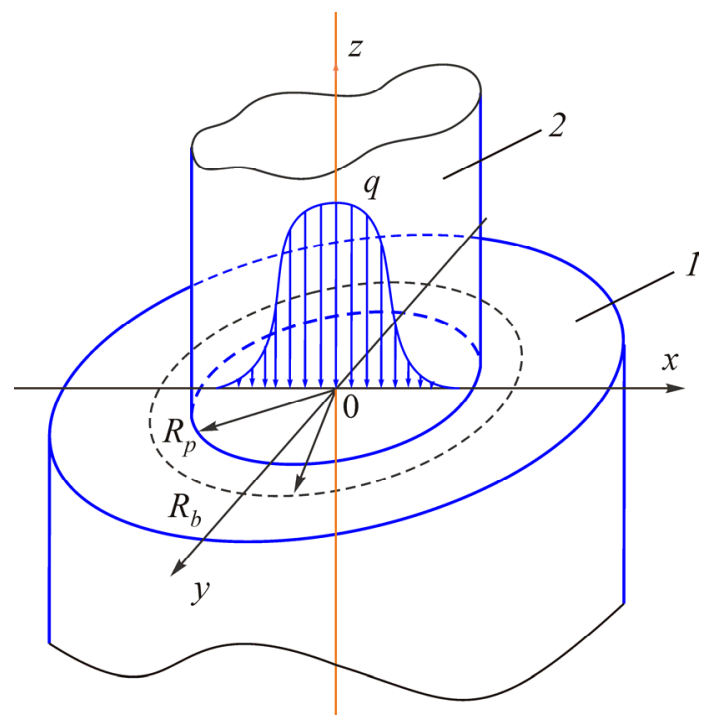

Рис. 1. Расчетная схема воздействия канала плазмы на поверхность обрабатываемой детали: $q$ - тепловой поток; 1 - электрод-деталь из стали; 2 - плазменный канал; $R_{p}$ - радиус источника тепла; $R_{b}$ - радиус газового пузыря

Уравнение непрерывности имеет вид

$$
\left(\frac{\delta \rho_{0}}{\delta t}\right)+\frac{1}{r} \frac{\delta}{\delta r}\left(\rho_{0} r v_{r}\right)=0
$$

где $\rho_{0}$ - плотность диэлектрика; $t$ - время; $r$ - радиус-вектор рассматриваемой точки; $v_{r}$ - скорость расширения канала плазмы.

Тогда скорость расширения канала плазмы описывается уравнением

$$
v_{r}=\left(\frac{R_{p}}{r}\right)\left(\frac{d R_{p}}{d t}\right),
$$

где $R_{p}$ - радиус канала плазмы.

Энергетический баланс для нестационарного состояния плазмы как для термодинамической системы имеет вид

$$
U I F_{p}=(25 I)(0,74)=\left(H-H_{0}\right)\left(\frac{d m}{d t}\right)+m\left(\frac{d H}{d t}\right),
$$

где $U$ - напряжение; $F_{p}$ - часть мощности, приходящейся на плазму; $H$ - средняя энтальпия плазмы; $H_{0}$ - энтальпия окружающего изолятора; $m$ - масса плазмы.

Ввиду короткого времени импульса (мкс) теплопередача в жидкости в канале пробоя посредством теплопроводности и конвекции незначительна.

Исходя из уравнения скорости расширения плазменного канала и энергетического баланса для нестационарного состояния плазмы, при создании математической модели необходимо задать следующие исходные параметры: энергия $-10^{6}$ Вт; приближенный радиус плазменного канала - 0,2 мм.

Подбор материалов для геометрических моделей осуществлялся из библиотеки Comsol Multiphysics. В связи с тем, что легирующие элементы стали 38Х2Н2MА влияют на ее критические точки, в теоретическую модель был внесен поправочный коэффициент.

Граничные условия модели определялись по методике, представленной в работах $[3,7]$ (рис. 2).

На границах $A B$ задаем условие осевой симметрии $(r=0)$. 
Границы $C D$ и $D E$ обрабатываемой детали омываются рабочей жидкостью (РЖ). Свойства рабочей жидкости, заполняющей межэлектродный зазор, оказывают большое влияние на формирование и развитие канала разряда. Диэлектрические свойства рабочей жидкости определяют величину пробивного зазора при заданной амплитуде напряжения и, следовательно, соотношение между энергией, выделяющейся на электродах. Чем выше диэлектрические свойства среды, тем меньше величина зазора, выше эффект полярности, хуже условия удаления продуктов эрозии из рабочей зоны. Условие непрерывности РЖ принимает вид

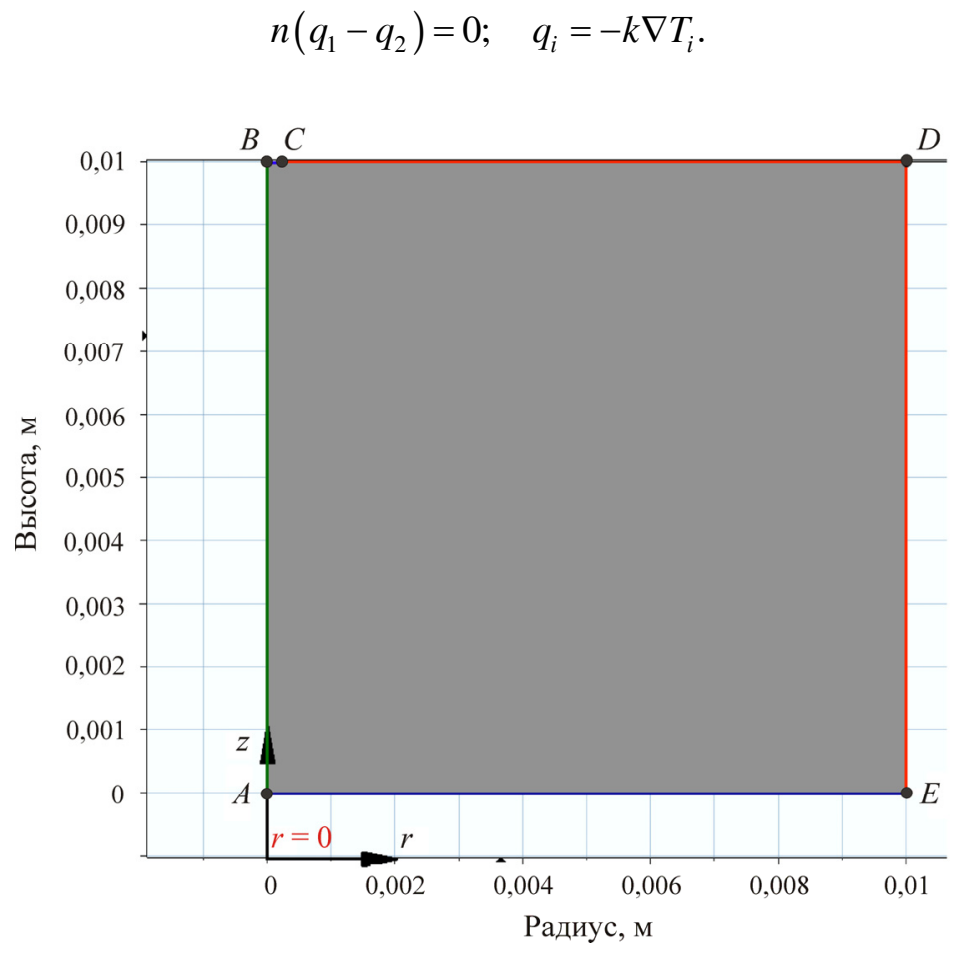

Рис. 2. Граничные условия

Высокотемпературный канал пробоя излучает энергию, которая поглощается окружающим диэлектриком так же, как и металлическими электродами. Условие термоизоляции на границах $A E$, через которые тепло не будет распространяться в окружающую среду, имеет вид

$$
-n(-k \nabla T)=0 .
$$

Граница $B C$ определяет зону воздействия плазменного канала, где воздействует тепловой поток $q$. Распределение потока $q$ проходит по нормальному закону распределения (закону Гаусса).

Величина теплового потока $q$ определяет количество тепла, проходящего через зону воздействия источника.

Взаимосвязь между величиной теплового потока и параметрами рабочего импульсного сигнала можно охарактеризовать следующей формулой:

$$
q(t)=\eta \frac{4(I(t) U(t))}{\pi R_{s}^{2}},
$$

где $\eta$ - доля энергии разряда, поступающая в исследуемый образец; $R_{s}$ - радиус канала разряда; $I(t)$ - функция силы тока $I$, А, в единицу времени $t ; U(t)$ - функция напряжения $U$, В, в единицу времени $t$.

Уравнение, описывающее характер распределения теплового потока по координате $x$, имеет вид 


$$
q(x)=\frac{1}{\sigma(t) \sqrt{2 \pi}} \cdot e^{\frac{-(x-m x)^{2}}{2 \sigma^{2}(t)}},
$$

где $\sigma$ - среднеквадратическое отклонение; $m x$ - математическое ожидание; $x$ - случайная величина.

После преобразования уравнений (1) и (2) уравнение теплового потока приобретает следующий окончательный вид:

$$
q(x, t)=\eta \frac{4(I(t) U(t))}{\pi R_{s}^{2}} \cdot \frac{1}{\sigma(t) \sqrt{2 \pi}} \cdot \exp ^{\frac{-(x-m x)^{2}}{2 \sigma^{2}(t)}} .
$$

Импульсное воздействие тока на поверхность заготовки учитывается путем использования функции Step. Введение в систему уравнений функции шага (Step) позволяет учитывать время действия импульса $T_{\text {on }}$ в канале разряда.

Плоский источник нагрева задается коэффициентом общего входящего теплового потока (general inward heat flux) во вкладке Heat Flux (cathode):

$$
q_{0}=\text { heatflux }(r) \cdot T_{o n}(t),
$$

где heatflux $(r)$ - функция нормального распределения величины теплового потока по координате $r$ при значении времени $t$, Вт/м ${ }^{2} ; T_{o n}(t)$ - шаговая функция времени действия импульса.

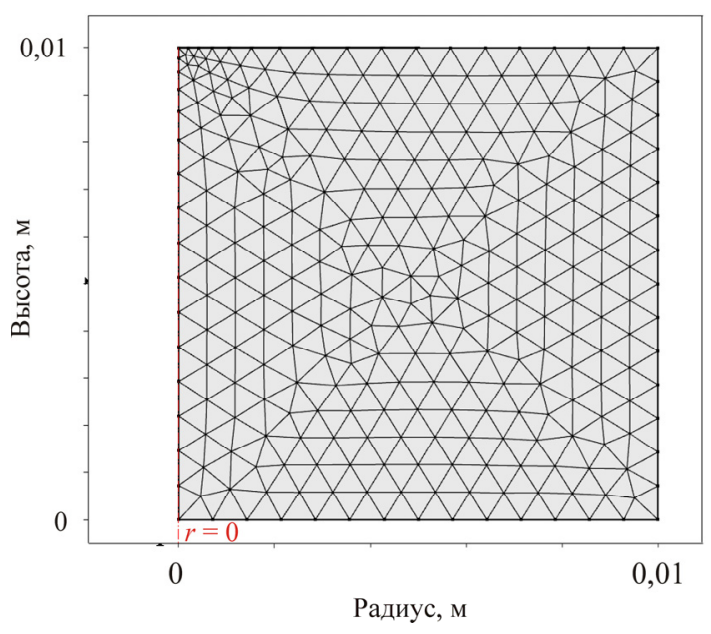

Рис. 3. Построение сетки конечных элементов

Построение сетки конечных элементов осуществляется при помощи функции Mesh (рис. 3).

Максимальный размер ячейки составляет $7,41^{-4}$ м; минимальный $-2,5^{-6}$ м.

\section{Результаты моделирования}

По результатам моделирования построен график изменения температуры на поверхности детали при ЭЭО (рис. 4).

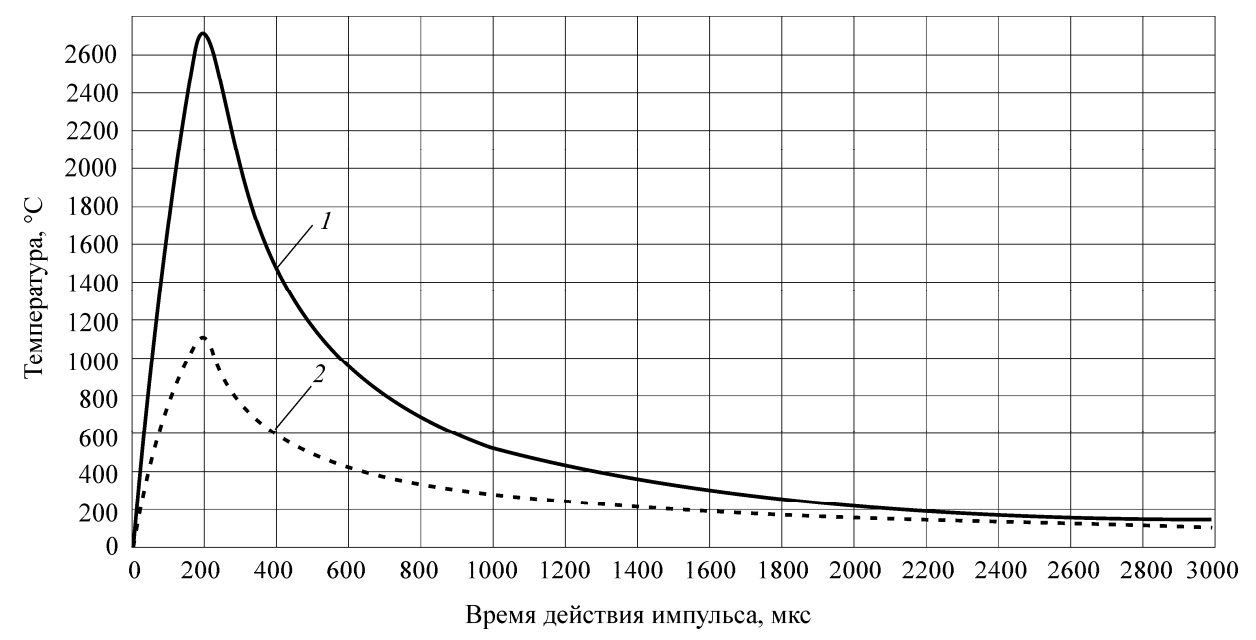

Рис. 4. График изменения температуры на поверхности детали в результате воздействия импульса: 1 - температура на поверхности детали в центре разряда; 2 - температура на поверхности детали на границе радиуса действия плазмы 
Анализируя график (см. рис. 4), можно сделать вывод о том, что при воздействии единичного разряда температура на поверхности обрабатываемой детали достигает максимального значения при $t=200$ мкс, металл при этом плавится и частично испаряется, оставляя за собой кратер (максимальная температура - рис. 6). После окончания действия теплового потока процесс продвижения границ плавления и испарения может некоторое время продолжаться за счет тепловой энергии, содержащейся в жидкой фазе, которая может иметь температуру, превышающую температуру плавления, а поверхностные слои могут иметь температуру, превышающую температуру кипения.

Анализ результатов расчета показал (рис. 5-7), что при воздействии единичного импульса величина единичной лунки зависит от энергии и времени действия импульса. Показано, что лунка имеет чашеобразную форму, т.е. ширина кратера выше по сравнению с глубиной, что не противоречит существующим научным данным [1-7]. Ширина и глубина кратера в диапазоне времени $t=100 \ldots 200$ мкс увеличиваются. При достижении времени $t=200$ мкс температура в лунке становится пиковой, затем она понижается.

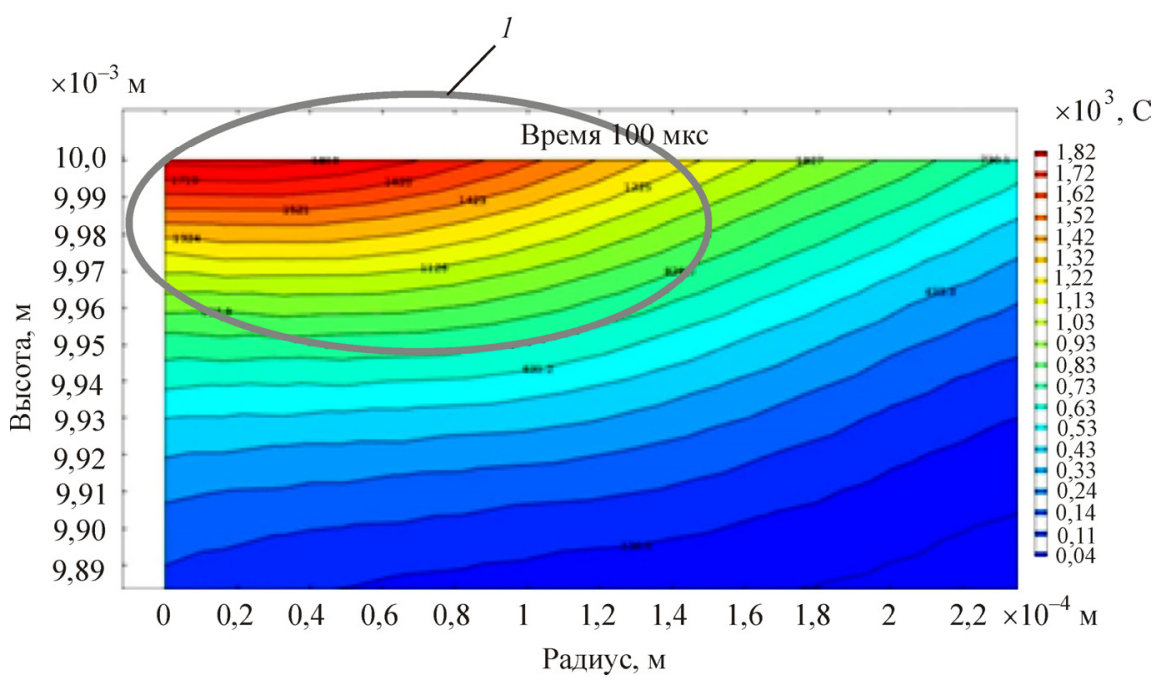

Рис. 5. Изменение температурных полей внутри ЭД при воздействии единичного разряда в момент времени $t=100$ мкс: $1-$ формирование единичной лунки

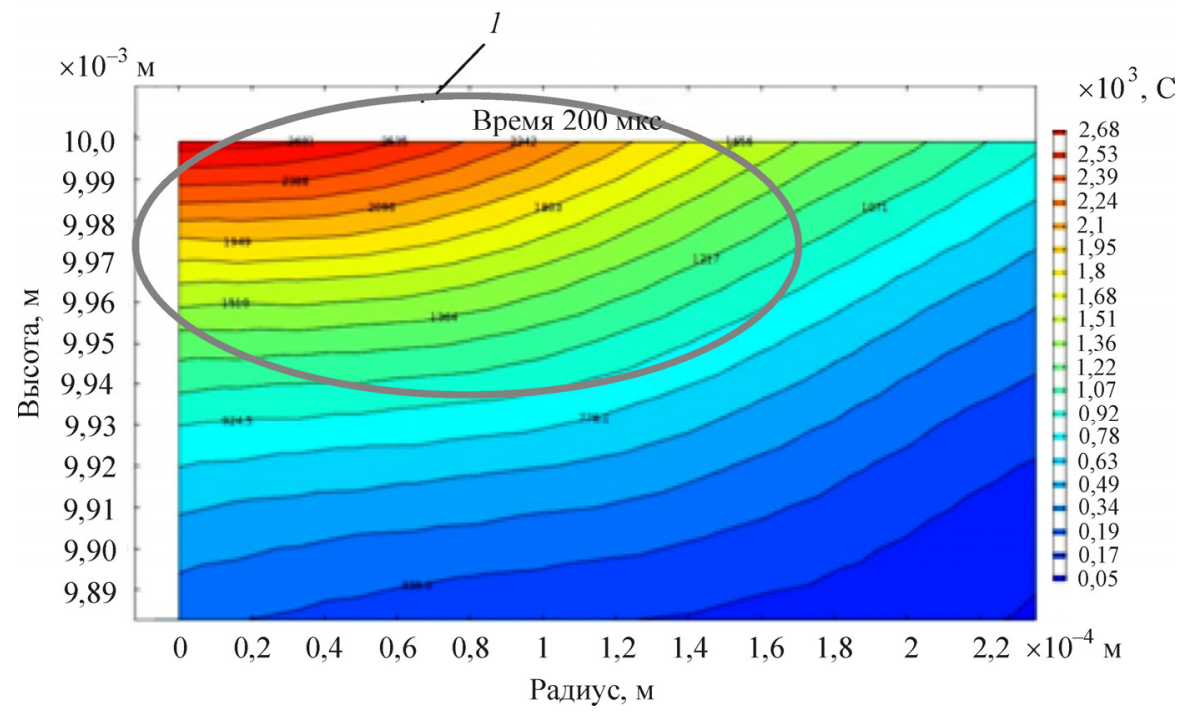

Рис. 6. Изменение температурных полей внутри ЭД при воздействии единичного разряда в момент времени $t=200$ мкс: $1-$ формирование единичной лунки 


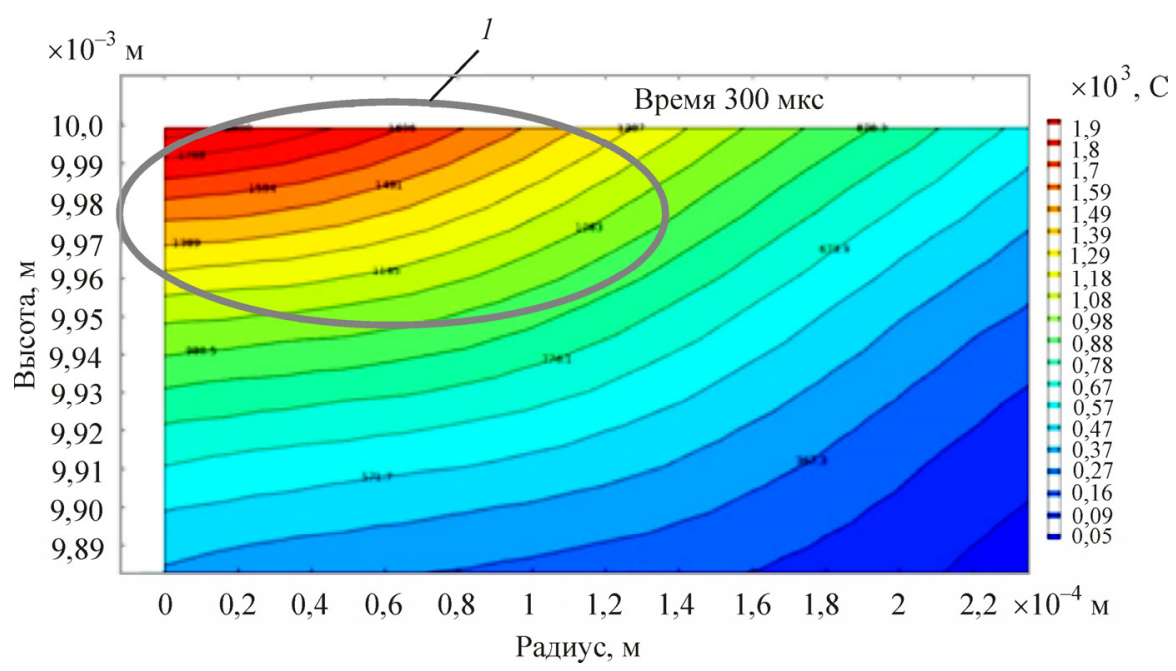

Рис. 7. Изменение температурных полей внутри ЭД при воздействии единичного разряда в момент времени $t=300$ мкс: $1-$ формирование единичной лунки

\section{Заключение}

Получена математическая модель, позволяющая описать величину температурного воздействия на поверхность заготовки при электроэрозионной обработке. Показано, что в результате электроэрозионной обработки температура на поверхности детали в центре разряда превышает $2500^{\circ}$, в результате чего на обработанной поверхности формируется единичная лунка.

Показано, что для повышения точности моделирования температурного воздействия на поверхность заготовки при ЭЭО необходимо учитывать импульсный характер воздействия тока и вводить в систему уравнений функцию Step.

Для уточнения полученных результатов моделирования необходимо провести серию экспериментальных исследований.

Исследования выполнены при финансовой поддержке Министерства образования и науки РФ по государственному заданию 11.9716.2017/8.9.

\section{Список литературы}

1. Абляз Т.Р., Ханов А.М., Хурматуллин О.Г. Современные подходы к технологии электроэрозионной обработки материалов. - Пермь: Изд-во Перм. нац. исслед. политехн. ун-та, 2012. - 112 с.

2. Серебреницкий П.П. Современные электроэрозионные технологии и оборудование: учеб. пособие / Балт. гос. техн. ун-т. - СПб., 2007. - 228 с.

3. Журин А.В. Методы расчета технологических параметров и электродов-инструментов при электроэрозионной обработке: дис. ... канд. техн. наук / Тул. гос. ун-т. - Тула, 2005. - 132 с.

4. Zlygostev A.M. The role of the plasma channel and the mechanisms of heat transfer in spark processing // Proceedings of KnAGTU. - 2011. - № II-1 (6). - P. 121.

5. Панов Д.О., Абляз Т.Р., Абросимова А.А. Металлографический анализ поверхности стали 65Г после электроэрозионной обработки [Электронный ресурс] // Современные проблемы науки и образования. - 2013. - № 5. - 6 c. - URL: http://www.science-education.ru/ru/article/view?id=10218 (дата обращения: 15.01.2018).

6. Абляз Т.Р., Симонов М.Ю., Шлыков Е.С. Анализ поверхности наплавленной меди после электроэрозионной обработки // Металловедение и термическая обработка металлов. - 2017. - № 12(750). C. 37-43.

7. Курочкин А.И. Обоснование условий и режимов высокочастотной микроэлектроэрозионной обработки: дис. ... канд. техн. наук / Тул. гос. ун-т. - Тула, 2016. - 139 с. 
8. Determination of the cathode erosion and temperature for the phases of high voltage discharges using FEM simulations / F. Soldera, A. Lasagni, F. Mücklich, T. Kaiser, K. Hrastnik // Computational Materials Science. - 2005. - № 32/1. - P. 123-139.

9. Marafona J., Chousal J.A.G. A finite element model of EDM based on the Joule effect // International Journal of Machine Tools and Manufacture. - 2006. - № 46/6. - P. 595-602.

10. Allen P., Chen X. Process simulation of micro electro-discharge machining on molybdenum // Journal of Materials Processing Technology. - 2007. - № 186. - P. 346-355.

11. Tan P.C., Yeo S.H. Modelling of overlapping craters in micro-electrical discharge machining // Journal of Physics D: Appliad Physics. - 2008. - № 41. - P. 205-302.

12. Thermal modeling of the mateail removal rate of high-speed small hole EDM drilling using the computational fluid dynamics method / Y. Wang, J. Bai, Y. Guo, H. Huang, J. Liu // Proceedings 16th International Symposium on Electromachining. - 2010. - P. 705-710.

13. Съянов С.Ю. Технологическое обеспечение качества поверхностного слоя деталей при электроэрозионной обработке: дис. .... канд. техн. наук / Брян. гос. техн. ун-т. - Брянск, 2002. - 166 с.

\section{References}

1. Abliaz T.R., Khanov A.M., Khurmatullin O.G. Sovremennye podkhody k tekhnologii elektroerozionnoi obrabotki materialov [Modern approaches to technology of electroerosive processing of materials]. Izdatel'stvo Permskogo natsional'nogo issledovatel'skogo politekhnicheskogo universiteta, 2012, $112 \mathrm{p}$.

2. Serebrenitskii P.P. Sovremennye elektroerozionnye tekhnologii i oborudovanie [Modern electroerosive technologies and equipment]. Baltiiskii gosudarstvennyi tekhnicheskii universitet. Saint-Petersburg, 2007, $228 \mathrm{p}$.

3. Zhurin A.V. Metody rascheta tekhnologicheskikh parametrov i elektrodov-instrumentov pri elektroerozionnoi obrabotke [Methods of calculation of technological parameters and electrodes tools at electroerosive processing]. Ph. D. thesis. Tula, 2005, 132 p.

4. Zlygostev A.M. The role of the plasma channel and the mechanisms of heat transfer in spark processing. Proceedings of KnAGTU, 2011, no. II-1 (6), p. 121.

5. Panov D.O., Abliaz T.R., Abrosimova A.A. Metallograficheskii analiz poverkhnosti stali $65 \mathrm{G}$ posle elektroerozionnoi obrabotki [The metalgraphic analysis of a surface of steel $65 \mathrm{G}$ after electroerosive processing]. Sovremennye problemy nauki $i$ obrazovaniia, 2013, no. 5, 6 p. Available at: http://www.scienceeducation.ru/ru/article/view?id=10218 (accessed 15 January 2018).

6. Abliaz T.R., Simonov M.Iu., Shlykov E.S. Analiz poverkhnosti naplavlennoi medi posle elektroerozionnoi obrabotki [The analysis of a surface of the built-up copper after electroerosive processing]. Metallovedenie i termicheskaia obrabotka metallov, 2017, no. 12(750), pp. 37-43.

7. Kurochkin A.I. Obosnovanie uslovii i rezhimov vysokochastotnoi mikroelektroerozionnoi obrabotki [Justification of conditions and modes of high-frequency microelectroerosive processing]. Ph.D thesis. Tula, 2016, 139 p.

8. Soldera F., Lasagni A., Mücklich F., Kaiser T., Hrastnik K. Determination of the cathode erosion and temperature for the phases of high voltage discharges using FEM simulations. Computational Materials Science, 2005, no. 32/1, pp. 123-139.

9. Marafona J., Chousal J.A.G. A finite element model of EDM based on the Joule effect. International Journal of Machine Tools and Manufacture, 2006, no. 46/6, pp. 595-602.

10. Allen P., Chen X. Process simulation of micro electro-discharge machining on molybdenum. Journal of Materials Processing Technology, 2007, no. 186, pp. 346-355.

11. Tan P.C., Yeo S.H. Modelling of overlapping craters in micro-electrical discharge machining. Journal of Physics D: Appliad Physics, 2008, no. 41, pp. 205-302.

12. Wang Y., Bai J., Guo Y., Huang H., Liu J. Thermal modeling of the mateail removal rate of highspeed small hole EDM drilling using the com-putational fluid dynamics method. Proceedings 16th International Symposium on Electromachining, 2010, pp. 705-710.

13. S"ianov S.Iu. Tekhnologicheskoe obespechenie kachestva poverkhnostnogo sloia detalei pri elektroerozionnoi obrabotke [Technological support of quality of a blanket of details at electroerosive processing]. Briansk, 2002, 166 p.

Получено 8.02.18 


\section{Об авторах}

Абляз Тимур Ризович (Пермь, Россия) - кандидат технических наук, доцент, старший научный сотрудник кафедры материалов, технологий и конструирования машин Пермского национального исследовательского политехнического университета; e-mail: lowrider11-13-11@ mail.ru.

Муратов Карим Равилевич (Пермь, Россия) - кандидат технических наук, доцент кафедры материалов, технологий и конструирования машин Пермского национального исследовательского политехнического университета; e-mail: lowrider11-13-11@mail.ru.

Красновский Евгений Ефимович (Пермь, Россия) - кандидат технических наук, доцент, доцент кафедры прикладной математики Московского государственного технического университета им. Н.Э. Баумана (национального исследовательского университета); e-mail: ee_krasnovskiy@mail.ru.

Борисов Денис Андреевич (Пермь, Россия) - магистрант кафедры материалов, технологий и конструирования машин Пермского национального исследовательского политехнического университета; e-mail: lowrider11-13-11@mail.ru.

\section{About the authors}

Timur R. Ablyaz (Perm, Russian Federation) - Ph. D. in Technical Sciences, Associate Professor, Senior Researcher, Department of Materials, Technologies and Constructions of Machines, Perm National Research Polytechnic University; e-mail: lowrider11-13-11@mail.ru.

Karim R. Muratov (Perm, Russian Federation) - Ph. D. in Technical Sciences, Associate Professor, Department of Materials, Technologies and Constructions of Machines, Perm National Research Polytechnic University; e-mail: lowrider11-13-11@ mail.ru.

Evgeny E. Krasnovsky (Moscow, Russian Federation) - Ph. D. in Technical Sciences, Associate Professor, Department of Applied Mathematics, Bauman Moscow State Technical University (National Research University of Technology); e-mail: ee_krasnovskiy@mail.ru.

Denis A. Borisov (Perm, Russian Federation) - Master Student, Department of Materials, Technologies and Constructions of Machines, Perm National Research Polytechnic University; e-mail: lowrider11-13$11 @$ mail.ru. 